\title{
Density Functional Theory Half Electron Self-Energy Correction for Fast and Accurate Nonadiabatic Molecular Dynamics
}

\author{
Yonghao Zhu ${ }^{1}$, Run Long ${ }^{1 *}$ \\ ${ }^{1}$ College of Chemistry, Key Laboratory of Theoretical \& Computational
}

Photochemistry of Ministry of Education, Beijing Normal University, Beijing, 100875,

$$
\text { P. R. China }
$$

\section{Density Functional Theory Half Electron Self-Energy Correction Method}

The concept of DFT-1/2 electron self-energy correction, including both LDA-1/2 and GGA-1/2, originates from Slater's half occupation scheme designed for ionization energy calculation for isolated atoms. According to the theorem proposed by Janak, ${ }^{1}$ the single particle eigenvalue, $e_{\alpha}$, is the variation of the total energy, $E$, with respect to its occupation, $f_{\alpha}$ :

$$
\frac{\partial E}{\partial f_{\alpha}}=e_{\alpha}\left(f_{\alpha}\right)
$$

There is a linear relationship between eigenvalue $\left(e_{\alpha}\right)$ and its occupation $\left(f_{\alpha}\right)^{2}$ Therefore, we can obtain:

$E(0)-E(-1)=e_{\alpha}(0)-S_{\alpha}$

where $E(0)$ and $E(-1)$ are the energy of ground and ion states (one electron is removed from state $\alpha$ ), respectively. $S_{\alpha}$ is named "self-energy" term with an analytical expression:

$S_{\alpha}=\iint d^{3} \boldsymbol{r} d^{3} \boldsymbol{r}^{\prime} \frac{n_{\alpha}(\boldsymbol{r}) n_{\alpha}\left(\boldsymbol{r}^{\prime}\right)}{\left|\boldsymbol{r}-\boldsymbol{r} \boldsymbol{\prime}^{\prime}\right|}+$ higher order terms

where $n_{\alpha}$ is the local electron density. The self-energy may be thought of as the quantum mechanical average of a "self-energy potential", $V_{s}$ :

*Corresponding author, E-mail: runlong@bnu.edu.cn 
$S_{\alpha}=\int d^{3} \boldsymbol{r} n_{\alpha}(\boldsymbol{r}) V_{s}(\boldsymbol{r})$

The $V_{s}(\boldsymbol{r})$ can be obtained as the difference from the DFT-calculated electrostatic potentials between the neutral atom and the half-ionized ions:

$V_{s}(\boldsymbol{r}) \approx-V\left(-\frac{1}{2}, \boldsymbol{r}\right)+V(0, \boldsymbol{r})$

The self-energy potential must be trimmed by a function, $\Theta(r)$ :

$\Theta(r)= \begin{cases}{\left[1-\left(\frac{r}{C U T}\right)^{n}\right]^{3},} & r \leq C U T \\ 0, & r>C U T\end{cases}$

The trimmed self-energy potential $\Theta(r) V_{S}(\boldsymbol{r})$ is entirely contained inside a sphere of radius CUT. The potential is added to the pseudopotentials of the anions that serve as external potentials. ${ }^{3}$ 


\section{Kinetic Models}

The schematics and basis set of the nonradiative electron-hole recombination for the rutile $\mathrm{TiO}_{2}$ and black phosphorus (BP) monolayer are shown in Figure S1. The valence band maximum (VBM) and conduction band minimum (CBM) correspond to the ground (GS) and excited states (ES), respectively. The population growth of the GS is shown in Figure S7. Fits of the data in Figure S7 using the short-time linear approximation of the exponential functional, $P(t)=\exp \left(-\frac{t}{\tau}\right) \approx 1-t / \tau$, gives the transition rates $\mathrm{k}$ between the $\mathrm{CBM}$ and VBM. The coupled kinetic equations for electron-hole recombination are as following:

$\frac{d[E S]}{d t}=-\mathrm{k}[\mathrm{ES}]$

$\frac{d[G S]}{d t}=k[E S]$

where $\mathrm{k},[\mathrm{ES}]$, and [GS] are the rate constant, the population of ES and GS, respectively. The solutions of above equations are:

$$
\begin{aligned}
& {[\mathrm{ES}]=e^{-k t}} \\
& {[\mathrm{GS}]=1-e^{-k t}}
\end{aligned}
$$

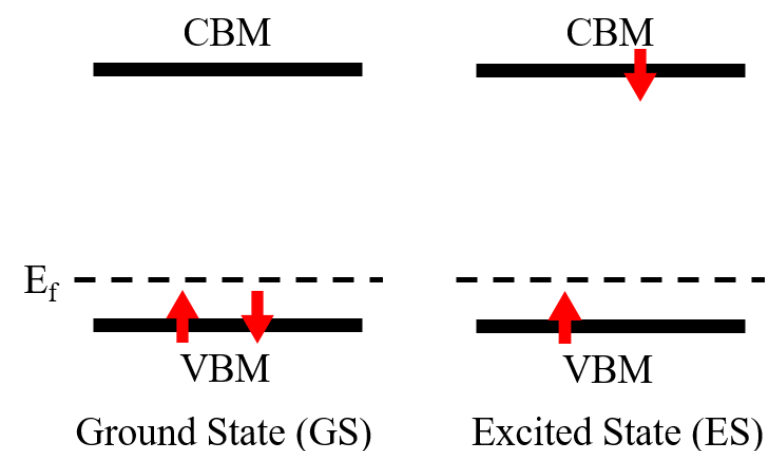

Figure S1. Schematics of electron-hole recombination in the bulk $\mathrm{TiO}_{2}$ and monolayer BP. 
(a) $\mathrm{TiO}_{2}$

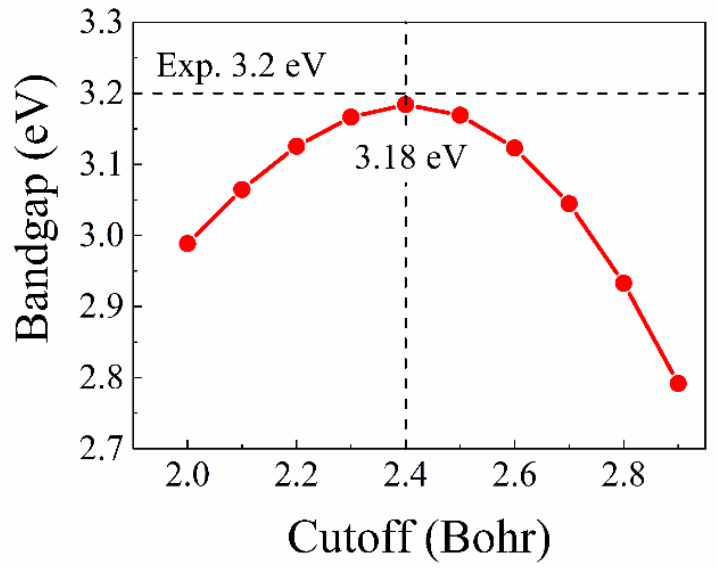

(b) BP

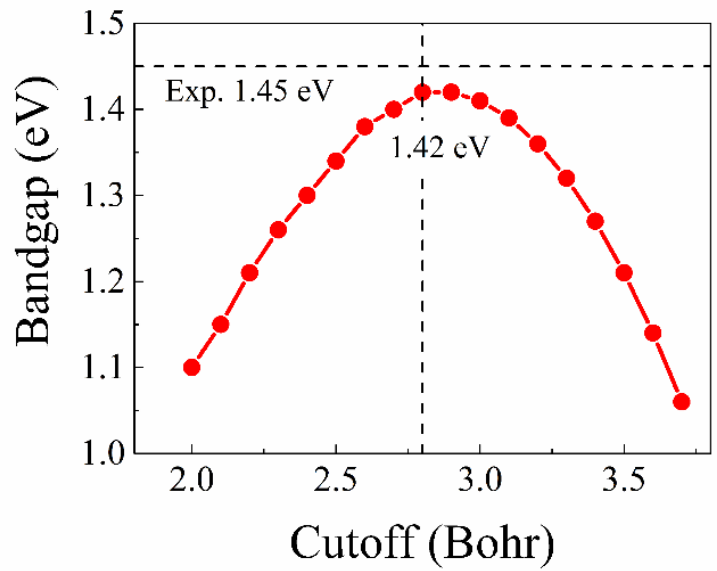

Figure S2. Variation of the bandgaps as function of the $\mathrm{O}$ and $\mathrm{P}$ cutoff radii in (a) bulk rutile $\mathrm{TiO}_{2}$ and (b) monolayer $\mathrm{BP}$, obtained using the PBE-1/2 method. ${ }^{4}$ 
(a) $\mathrm{TiO}_{2}$
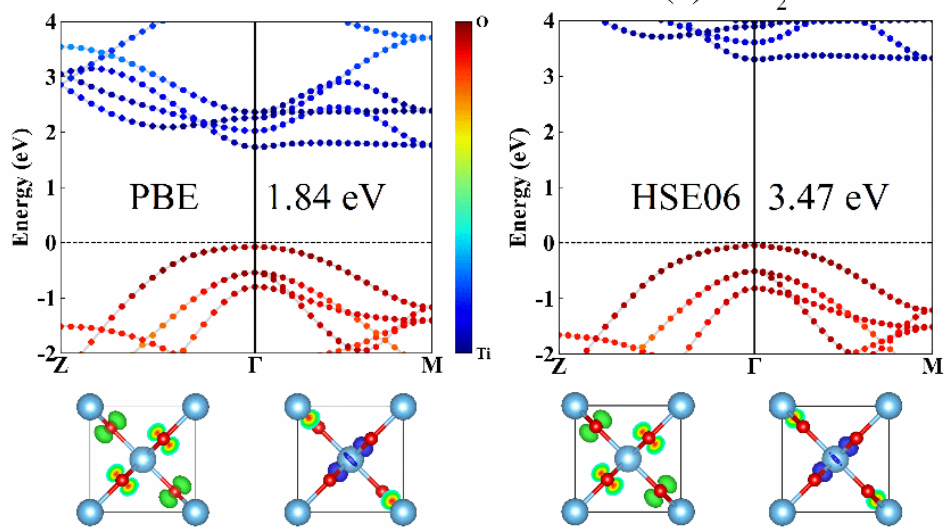

(b) $\mathrm{BP}$
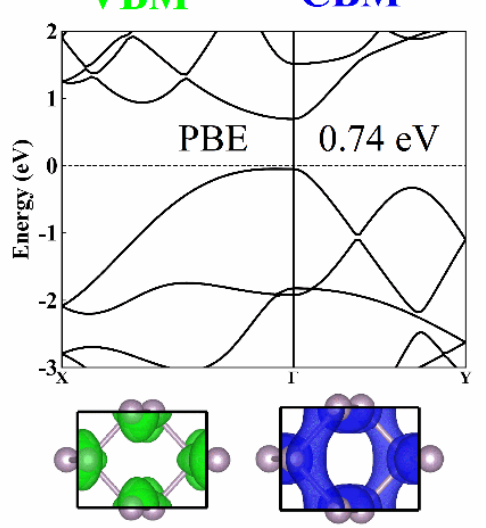
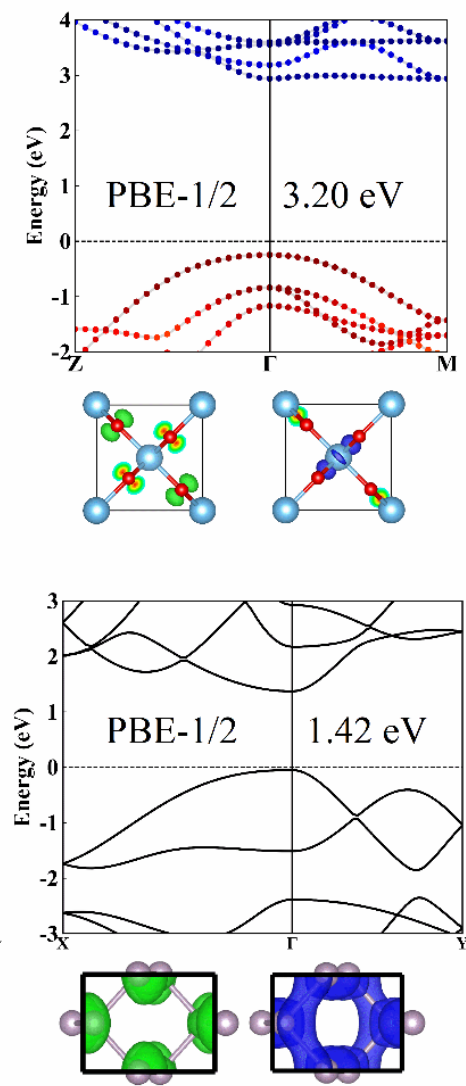

Figure S3. Band structure and the $\mathrm{CBM}$ and $\mathrm{VBM}$ in (a) $\mathrm{TiO}_{2}$ and (b) $\mathrm{BP}$ obtained using the PBE, HSE06 functionals, and PBE-1/2 method, respectively. The bandgaps produced by PBE-1/2 method agree well with the HSE06 functional. The charge densities remain almost same using the three methods. 


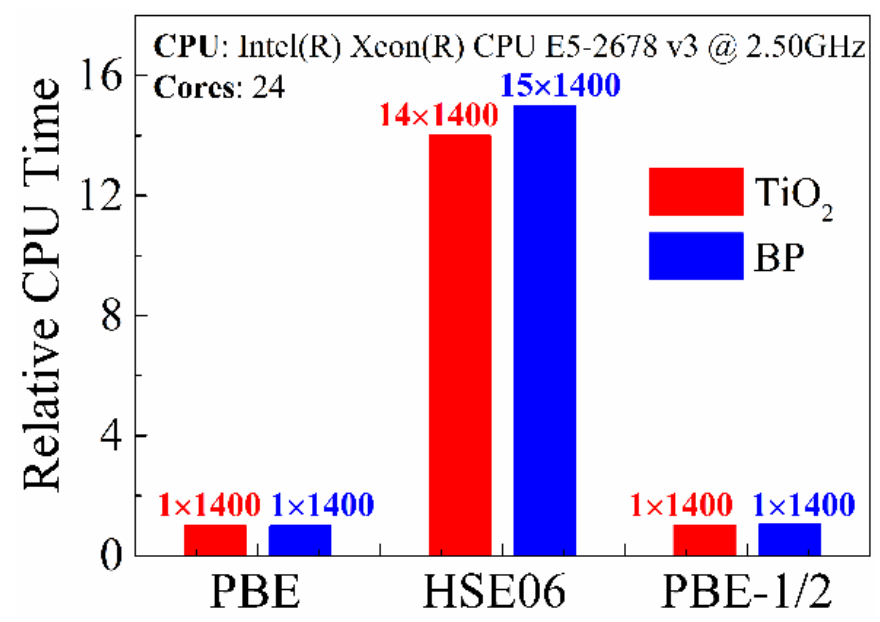

Figure S4. Relative central processing unit (CPU) times of 1400 nonadiabatic couplings calculations for $\mathrm{TiO}_{2}$ and $\mathrm{BP}$ using the PBE, HSE06, and PBE-1/2 methods. By setting the CPU time of PBE $\left(t_{P B E-s c f}^{a v g}\right)$ to 1 of one NA coupling for both $\mathrm{TiO}_{2}$ and BP systems, PBE-1/2 method leads to same CPU time and HSE06 functional increases time cost by 14 and 15 times ( $\left.n_{\text {times }}\right)$ than PBE functional for $\mathrm{TiO}_{2}$ and $\mathrm{BP}$, respectively. The total CPU time of overall NA couplings estimates as $t_{\text {total }} \approx N \times t_{s c f}^{a v g}$, where $N$ is the number of NA couplings. The difference in CPU time between HSE06 and PBE and PBE-1/2 methods can be written as $t_{\text {diff }} \approx N \times t_{P B E-s c f}^{a v g} \times n_{\text {times }}$. Here, $\mathrm{N}$ is 1400 in our current work, and $t_{P B E-s c f}^{a v g}\left(t_{P B E-1 / 2-s c f}^{a v g}\right)$ is about 25 seconds and 62 seconds for $\mathrm{TiO}_{2}$ and $\mathrm{BP}$, respectively. Therefore, the PBE-1/2 methods save about 3000 and 8100 cores $\times$ hours for $\mathrm{TiO}_{2}$ and $\mathrm{BP}$ with respect to HSE06 functional, respectively. 
(a) $\mathrm{TiO}_{2}$

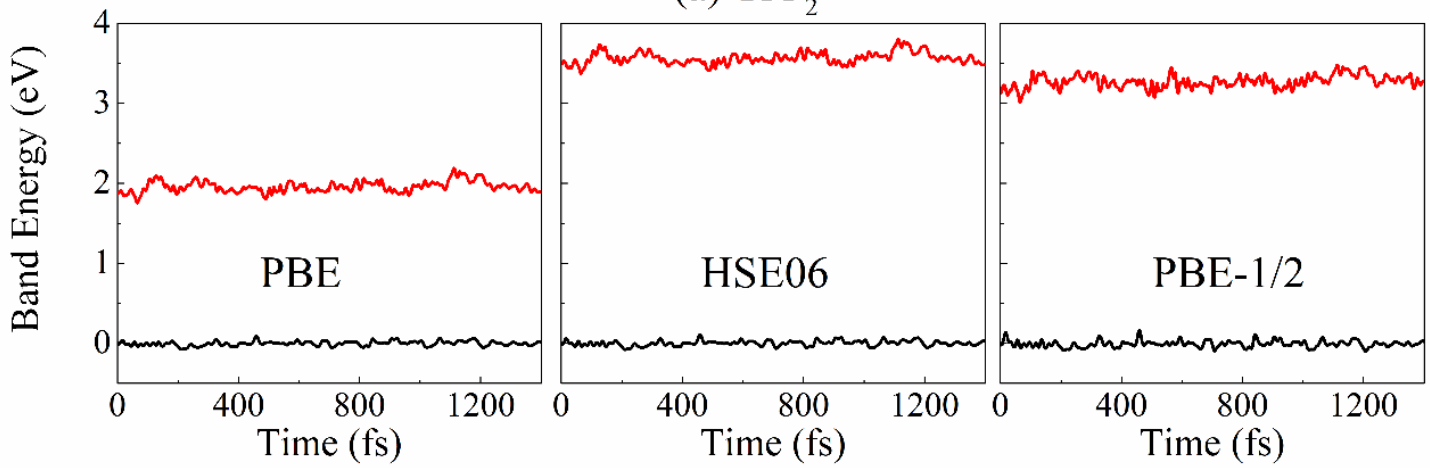

(b) BP

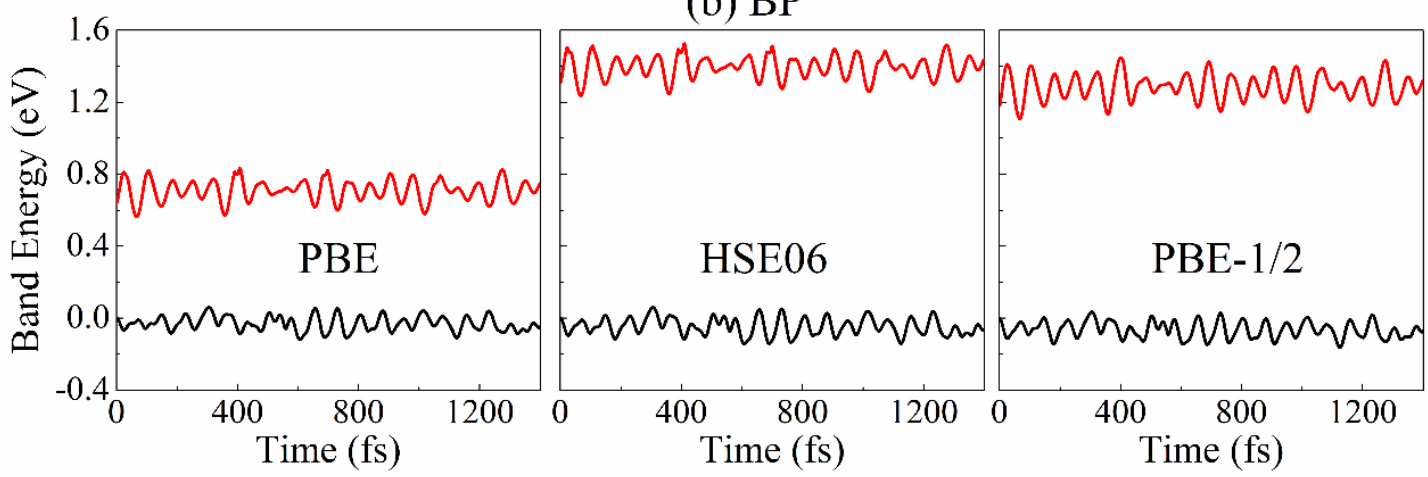

Figure S5. Time evolution orbital energies of the $\mathrm{CBM}$ and $\mathrm{VBM}$ in (a) rutile $\mathrm{TiO}_{2}$ and

(b) monolayer BP, calculated using the by PBE, HSE06, and PBE-1/2 methods, respectively. The fluctuations of orbital energies decrease from the PBE-1/2 method, to HSE06, to PBE functional. 
(a) $\mathrm{TiO}_{2}$

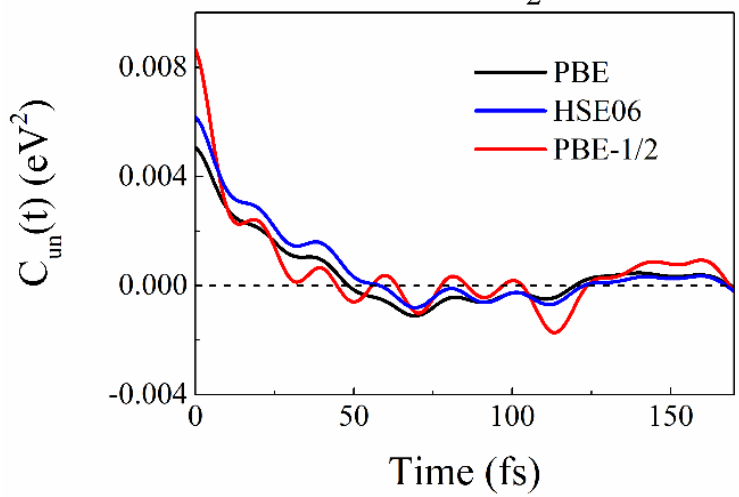

(b) BP

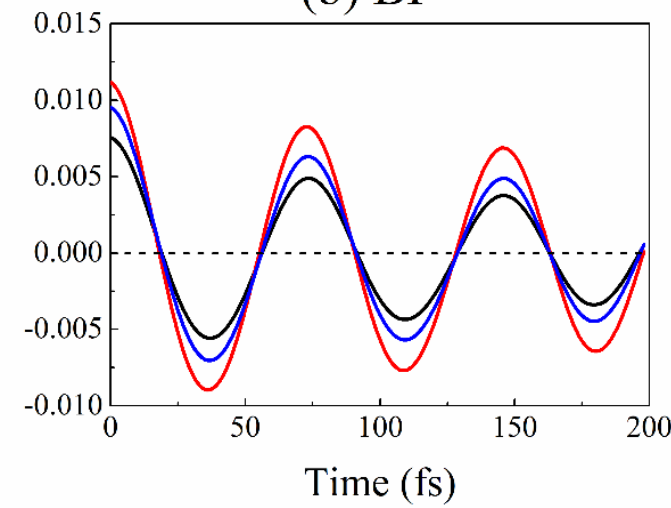

Figure S6. Unnormalized autocorrelation functions (un-ACF) of (a) $\mathrm{TiO}_{2}$ and (b) $\mathrm{BP}$. The black, blue, and red line represent the data obtained using the PBE, HSE06, and PBE-1/2 methods, respectively. In general, larger initial value of un-ACF leads to short pure-dephasing time. 
(a) $\mathrm{TiO}_{2}$
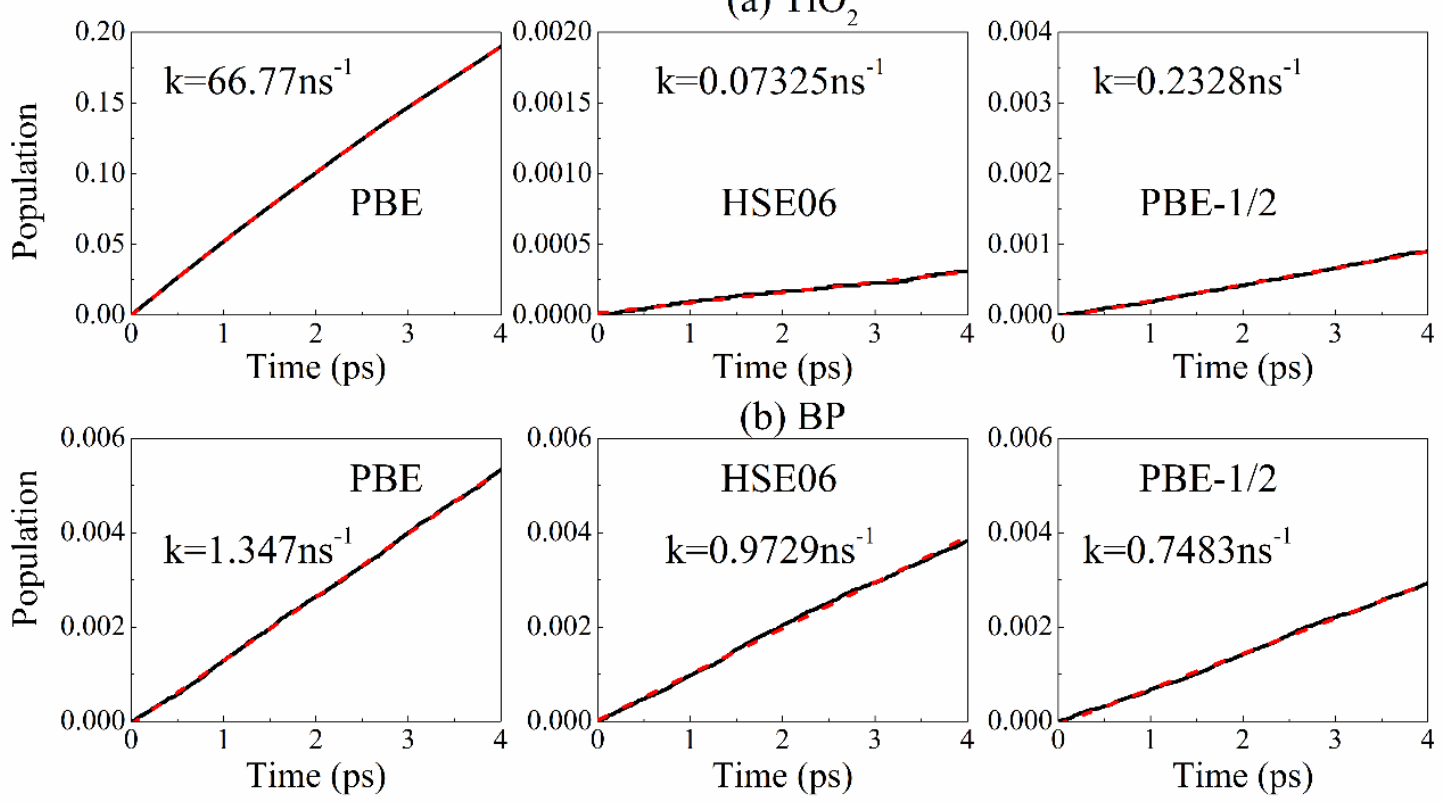

Figure S7. Time evolution of population of the ground states in (a) $\mathrm{TiO}_{2}$ and (b) $\mathrm{BP}$ using the NACs and energies obtained from the PBE, HSE06, and PBE-1/2 methods, respectively. The simulated data (black solid line) are fitted to (red dashed line) the short-time linear approximation to the exponential function, $P(t)=1-\exp (-t / \tau) \approx$ $t / \tau$. The inverse of times $\tau$ gives the rate constants in each panel. 
(a) $\mathrm{TiO}_{2}$

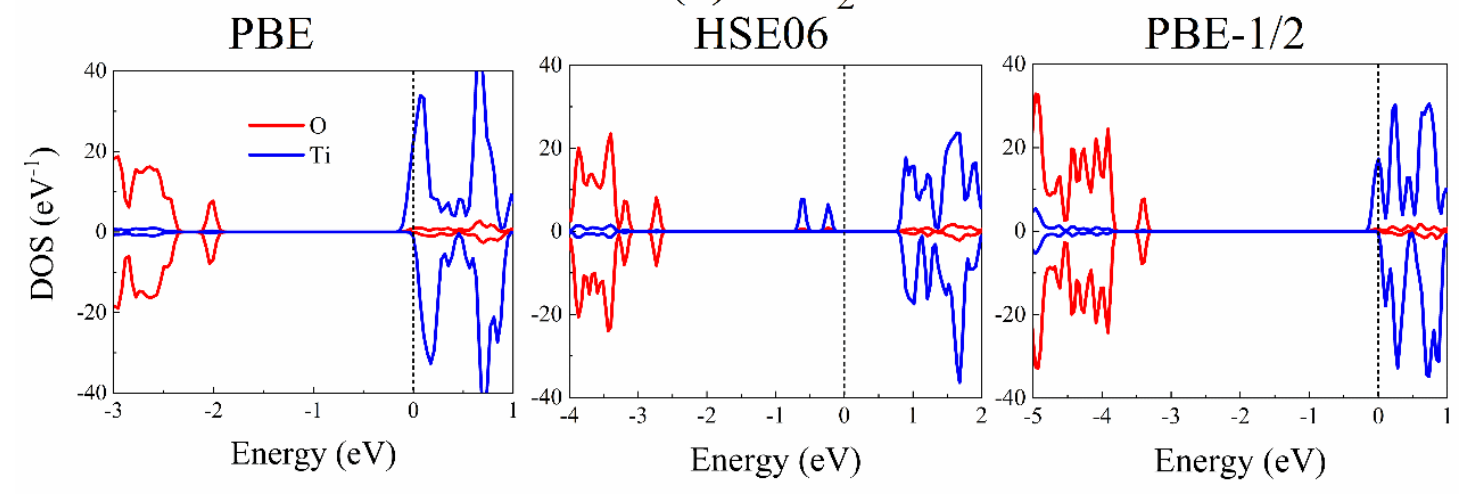

(b) BP
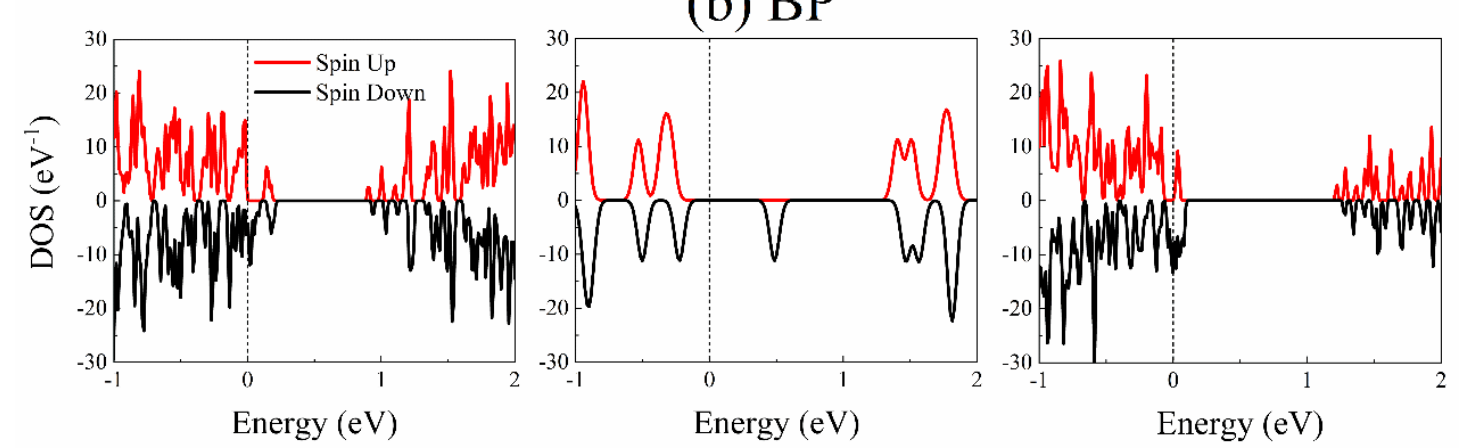

Figure S8. Projected density of states (PDOS) of (a) $\mathrm{TiO}_{2}$ containing a single oxygen vacancy and (b) monolayer BP containing a phosphorus vacancy, calculated using PBE, HSE06, and PBE-1/2 methods. 


\section{REFERENCES}

1. Janak, J. F. Proof that $\frac{\partial \mathrm{E}}{\partial \mathrm{n}_{\mathrm{i}}}=\varepsilon$ in density-functional theory. Phys. Rev. B 1978, 18, 7165-7168.

2. Leite, J. R.; Ferreira, L. G. Effects of the Coulomb Correlation on the Calculated Results for Atoms with and without Spin Polarization. Phys. Rev. A 1971, 3, 1224-1230.

3. Ferreira, L. G.; Marques, M.; Teles, L. K. Approximation to density functional theory for the calculation of band gaps of semiconductors. Phys. Rev. B 2008, 78, 125116.

4. Yuan, J.-H.; Chen, Q.; Fonseca, L. R. C.; Xu, M.; Xue, K.-H.; Miao, X.-S. GGA-1/2 self-energy correction for accurate band structure calculations: the case of resistive switching oxides. J. Comput. Phys. 2018, 2, 105005. 